\title{
Editorial
}

\section{The long and winding road of schizophrenia research}

\author{
ASSEN JABLENSKY
}

\section{INTRODUCTION}

Schizophrenia is a severely disabling disorder that occurs at comparable rates and with a strikingly similar clinical presentation in diverse populations (Jablensky $e t$ al., 1992). With a lifetime risk at about $1 \%$, it is estimated to account today for $2.6 \%$ of the global burden of disease (Murray \& Lopez, 1996). As far as historical records go, its incidence does not appear to have changed substantially over the past century. All of this suggests that the liability to the disorder is widely distributed and likely to be of ancient origin. The diagnostic concept of schizophrenia, originating with Kraepelin (1909) and Bleuler (1911), has proved remarkably robust despite recurrent attempts to revise or discard it, and has found its present formulation in the ICD-10 and DSM-IV diagnostic criteria.

It is now generally accepted that schizophrenia is a complex disorder with a major genetic contribution to its aetiology (heritability at about 0.80 ), probably involving multiple genes and locus heterogeneity (Riley et al., 2003). Environmental factors (ranging from neurodevelopmental insults to psychosocial factors) may interact with the genetic susceptibility to produce considerable variation in its phenotypic expression, which includes a proportion of transmitted genotypes that remain unexpressed as clinical disorder (Gottesman \& Bertelsen, 1989).

Address for correspondence: Professor A. Jablensky. School of Psychiatry and Clinical Neurosciences and Centre for Clinical Research in Neuropsychiatry. The University of Western Australia. MRF Building, 50 Murray Street. Perth WA 6000, Australia.

E-mail: assen@cyllene.uwa.edu.au

Declaration of Interest: none.

\section{VISION AND REALITY}

It is widely believed that the elucidation of the complex genetic basis of schizophrenia will lead to fundamental insights into its pathogenesis, novel therapeutic tools and preventive strategies. Indeed, research in schizophrenia has in the past two decades not only increased exponentially in volume, but has undergone a far-reaching qualitative change. Novel research technologies, ranging from molecular genetics to functional neuroimaging, are now center stage, tending to dislodge traditional clinical research and, to some extent, epidemiology. Following on the spectacular recent advances in molecular genetics, genomics and neuroscience, new cohorts of investigators are tempted by the vision that the unraveling of the causes of schizophrenia may be just around the corner. Yet a critical look at the balance sheet of the past two decades will reveal that no major breakthrough has yet occurred in key areas of schizophrenia research, such as the aetiology of the disorder, its risk factors, genetic basis and prevention. Recent reviews and meta-analyses of the genetic linkage and association findings point to multiple chromosomal regions that may harbour susceptibility genes. However, there are only 9 candidate genes, for which supportive evidence has been partially replicated (Harrison \& Owen, 2003) and no causative allele, or a genetic mutation, has to date been unequivocally demonstrated (Harrison \& Weinberger, 2005). No less ambiguous is the state of affairs in the neuroscience of schizophrenia, where progress appears to be constrained by the extreme individual variation in all phenotypic domains of the disorder, ranging from neuroanatomy to neuropsychology (Heinrichs, 2004). 


\section{DESIGN FLAWS AND CONCEPTUAL CONSTRAINTS}

Apart from the inherent complexity of the disorder, both methodological flaws and conceptual ambiguities complicate the search for replicable and coherent findings in schizophrenia research. First, there is the unfortunate tendency of selecting a single isolated feature - be it a symptom, a neuropsychological dysfunction, a neurotransmitter abnormality, a neuroanatomical marker, or a genetic polymorphism - and aiming to demonstrate a statistically significant difference (e.g. at $\mathrm{p}<0.05$ ) between its group mean value in what typically is a small sample of schizophrenia patients and in a comparison group (which is often recruited as a sample of convenience). The thorny issues of effect sizes and confidence intervals are rarely addressed and the feature may then be reported as vaguely implicated in the causation of the disorder. The output of this approach (illustrated by scores of journal publications) is a large number of non-replicable findings, which - seen in their totality - suggest that no brain structure, neurophysiological function, or genomic region is spared in schizophrenia. This approach inevitably leads to losing the "big picture" of the disease as a system and has produced over the last decades many short-lived "breakthroughs" in the understanding of schizophrenia, which have proved to be spurious.

Secondly, the prevailing paradigm in any domain of schizophrenia research - genetics, neurophysiology or neuroimaging - is still based on the implicit assumption that schizophrenia is a single, uniform disorder and that its ICD-10 or DSM-IV diagnosis identifies a biologically meaningful entity. However, an underlying aetiological heterogeneity, anticipated by Kraepelin and Bleuler, is likely to be a critical part of the explanation for the many non-replications of initially promising findings of genetic linkage and association studies conducted in recent years. Genetic heterogeneity is common in complex diseases and schizophrenia is unlikely to be an exception from the rule. What makes schizophrenia refractory to the available methods of dealing with heterogeneity is the confounding effect of a phenotype defined solely by a set of clinically conspicuous symptoms and behaviours. These clinical manifestations of schizophrenia may mask several underlying, different or partially overlapping, brain disorders whose pathogenesis and developmental history involves interactions of polygenes and environmental risk factors. Though many investigators and clinicians would readily subscribe to such a view, the diagnostic phenotype continues to be treated as a monolithic entity in the prevailing practices of schizophrenia research. This is likely to result in research collections of cases and families that contain admixtures of different variants of the disorder, where disparate 'biological signals' may cancel each other, yielding disappointingly small effect sizes for any variable of possible pathogenetic significance.

\section{LIMITATIONS OF THE CLINICAL PICTURE AS A PHENOTYPE FOR RESEARCH}

The clinical entity of schizophrenia appears to be a loose cluster of heterogeneous symptoms and traits, held together by a "deep structure" (Meehl, 1990), which at present eludes our understanding. There is a growing realization that the current ICD-10 and DSM-IV diagnostic definitions, based on heterogeneous subjective symptoms and behavioural deviance, do not provide the most appropriate phenotype for genetic or other aetiological research (Bearden et al. 2004). Several studies have refocused the phenotype definition on more homogeneous groups of clinical symptoms, obtained by clustering, factor-analytical or latent class techniques. An example is the delineation of a "deficit syndrome" in schizophrenia, based on selected negative symptoms presumed to be primary manifestations of the disease process (Carpenter $e t$ al., 1988). Such strategies of "splitting" or "lumping" diagnostic categories are a time-honoured approach in the search for biologically meaningful disease entities. However, many past attempts to re-package the clinical symptoms of schizophrenia into different factors or clusters have achieved little in terms of advancing aetiological research - possibly because symptoms such as delusions and hallucinations are complex subjective phenomena, far removed from the primary site of action of the biological causes.

\section{THE ENDOPHENOTYPE CONCEPT}

Other investigators have espoused Gottesman's concept (Gottesman \& Gould, 2003) of endophenotype (also termed correlated or intermediate phenotypes) as an alternative or a complement to the clinical diagnosis. Endophenotypes are heritable, objectively measurable biological traits, which co-segregate with clinical illness in pedigrees and may also be expressed in clinically unaffected members. They are stable, persist across clinical states and are likely to be more proximal to the primary biological defect and its genetic signal than the clinical phenotype. Examples of single endophenotypes, for which genetic linkage or association findings have been 
reported (though not adequately replicated), include event-related brain potentials (Freedman et al., 1997); smooth-pursuit eye movements (Arolt et al., 1996); and neuropsychological measures of executive dysfunction (Egan et al., 2001), learning and memory (Paunio et al., 2004).

In view of the variety of features that meet potential endophenotype criteria, the selection of informative endophenotypes for aetiological research becomes critical. A systematic effort at providing a rule-based evaluation of the evidence, using the tools of meta-analysis to estimate effect sizes (with confidence bounds) for a wide range of cognitive, neurophysiological and neuroimaging markers in schizophrenia, has been undertaken by Heinrichs \& Zakzanis (1998). This analysis demonstrates that the neurocognitive and neurophysiological measures (of memory, attention, executive function, sensory gating and general ability) are particularly sensitive to aspects of dysfunction that set schizophrenia patients apart from healthy controls. In terms of performance, these two classes of relatively low-cost measures are at present superior to the "high-tech" neuromorphological and neuroimaging markers and can we investigated with a greater return on informativeness in large samples.

Cognitive deficit as a core feature of schizophrenia was part of E. Kraepelin's original definition of dementia praecox as a disorder essentially characterised by "weakening of the mainsprings of volition", "lowered mental efficiency", "unsteadiness of attention", "inability to sift, arrange and correct ideas, and to accomplish mental grouping of ideas" (Kraepelin, 1909). Compromised higher cognitive function prior to the onset of disease has been shown to be a risk factor for schizophrenia in two large, population-based studies (David et al., 1997; Davidson et al., 1999). Schizophrenia patients exhibit abnormalities in multiple cognitive domains that predate the onset of disorder (Kremen et al., 1998; Bilder et al., 2000); persist across changes in the clinical state (Hoff $e t$ al., 1999); are not attributable to antipsychotic medication (Torrey, 2002); occur in non-psychotic relatives (Sitskoorn et al., 2004); and are specific to schizophrenia as compared to other psychotic disorders (Altshuler $e t$ al., 2004), thus meeting criteria for an endophenotype. The relevant cognitive indicators with moderate to high effect sizes include attention and memory deficits, executive dysfunction, and sensory gating (Heinrichs, 2004). It is, however, likely that most cognitive measures available today assess particular facets of complex interactive neural networks, rather than self-contained processing modules, and need to be investigated jointly (Phillips \& Silverstein, 2003).

\section{ONE DISORDER OR MANY?}

A major, yet rarely addressed question in schizophrenia research is: how to model in the study design the inherent phenotypic heterogeneity of the disorder? Fundamentally, two alternative (though not entirely irreconcilable) concepts are the view of schizophrenia as a unitary disorder and the hypothesis that the diagnostic category is a conflation of multiple disease variants or subtypes. According to the unitary view, schizophrenia is best conceptualised as one neurodevelopmental disorder where clinical heterogeneity is due to variable rates of pathological progression along multiple dimensions of brain structure and function. The alternative model of schizophrenia as a composite disease entity, originally foreshadowed by E. Bleuler (1911), posits several variant disorders, each underpinned by a relatively distinct aetiology and/or pathophysiology, but all sharing a broad, "common final pathway" of clinical phenotype expression (this model has well-known counterparts in mental retardation, dementia, or epilepsy). Clinical samples, selected solely on the broad diagnostic category, are predicted by this model to contain an admixture of different disorders, and the corollary is that parsing such samples into component subtypes will reduce biological heterogeneity. Substantial indirect support for this model is provided by the successful application of "splitting" strategies in the unraveling of the genetics of other complex diseases, such as diabetes or breast cancer.

The inherent heterogeneity, which was built into the original concept of schizophrenia, but became obfuscated in modern diagnostic classifications (DSM-IV and ICD10 ), is now coming back to centre stage as a working biological model. The meta-analytical findings (Heinrichs, 2004) support a reframing of the problem of excessive heterogeneity of research findings in schizophrenia as a problem of "parsing" the syndrome into several aetiologically distinct subtypes, using a combination of clinical, neurocognitive, neurophysiological and, possibly, neuroimaging measures - an objective that is being shared by an increasing number of investigators.

\section{STATISTICAL MODELING OF DISEASE HETEROGENEITY}

A difficult question in research design involving multi-domain measurements is how to treat statistically the many variables assessed. If the aim is to use multiple measurements of brain function (or dysfunction) in order to assess complex interactive neural networks, then what 


\section{A. Jablenshy}

is needed is means of identifying composite patterns of dysfunction, rather than single, unrelated deficits. Such composite interactive patterns should be capable of accounting for a greater proportion of the variance than the sum of individual components. One such analytical tool is the grade of membership (GoM) model. developed in the 1970s by Max Woodbury at the Center for Demographic Studies of Duke University, North Carolina (Woodbury et al.. 1978). GoM is not yet part of the "mainstream" statistical armamentarium, but it has been used in a growing number of studies, including research into schizophrenia. Alzheimer's disease, personality disorders and depression. Basically, GoM belongs in the family of latent structure analyses, introduced in the social sciences in the $1950 \mathrm{~s}$, and is closely related to latent class analysis (LCA). Like LCA, GoM partitions the data matrix into latent classes, called "pure types", by iteratively computing multiple regression relationships among all variables and estimating the maximum likelihood fit to alternative models consisting of varying numbers of pure types. Unlike LCA, where latent classes are defined as crisp partitions, GoM pure types are fuzzy sets, allowing their members to be simultaneously represented on more than one pure type by grade of membership coefficients. Thus, an individual may be described as either fully belonging to one pure type or partially to several. The attraction of the GoM model is that it mirrors the inherent "fuzziness" of psychiatric classification, where boundaries between syndromes tend to be poorly demarcated or may not exist at all.

\section{APPLICATION OF A HETEROGENEITY MODEL: THE WESTERN AUSTRALIAN FAMILY STUDY OF SCHIZOPHRENIA}

In a recently completed study of 112 families with members affected with schizophrenia and a general population control sample, we adopted a working model of schizophrenia as a phenomenological generic group, comprising several partially overlapping subtypes of underlying pathophysiology and causal pathways. GoM was employed as a tool for the detection of latent patterns of cognitive deficit in large datasets of neurocognitive measurements and personality attributes, obtained from patients, their first-degree relatives, and control subjects. The statistically most parsimonious solution yielded four pure types, of which two picked up the majority of patients with schizophrenia and a proportion of their clinically unaffected siblings or parents, while the remaining two mainly consisted of normal controls and relatives. On examination, these pure types represented different configurations and degrees of severity of cognitive deficits involving attention, executive functions, verbal memory and IQ, as well as personality traits, such as "positive" and "negative" schizotypy. magical ideation and harm avoidance.

We finally collapsed the three types into two contrasting groups - one with severe and pervasive cognitive deficit and another with less pronounced, patchy deficits but with marked deviations in the personality traits assessed. The patients falling into these two groups did not differ on duration of previous illness, which suggested that the two types were unlikely to represent different stages of progression of a single disease process. Moreover, the pattern of pervasive cognitive deficit showed significant familial aggregation (many of the clinically unaffected relatives exhibited attenuated deficits similar to those manifest in the patients), while the pattern of milder, patchy deficits did not. The identification of pervasive cognitive deficit as an endophenotype in some, but not all, patients and their family members suggested that it might characterise a genetically distinct schizophrenia subtype. To test this hypothesis, we conducted a whole genome scan, followed by linkage analysis including both diagnosis and neurocognitive profiles as a composite phenotype. The results (Hallmayer et al.. 2005) provide strong evidence that a variant or subtype of schizophrenia, characterised by generalized cognitive deficit, is linked to a narrow region on chromosome $6 \mathrm{p} 25-24$, which is likely to harbour a novel susceptibility gene of a relatively strong effect.

\section{CONCLUSIONS AND PROSPECTS}

The search for causative genes and pathogenetic pathways underlying schizophrenia has been hampered by a combination of factors, including its complex genetics, lack of "hard" phenotypic markers, the definition of the clinical phenotype solely by behavioural and psychopathological criteria, and, last but not least, absence of an established animal model. It is increasingly unlikely that our current diagnostic classifications based on symptoms, such as DSM-IV or ICD-10, "carve" nature at its genetic joints. The clinical manifestations of schizophrenia may represent a "common final pathway" for several different brain disorders (schizophrenia variants) with distinct genetic underpinnings. Therefore, the search for "specificity" of biological findings to schizophrenia as a clinically defined entity may be misguided - the clinical disorder, with its variable symptomatology and longitu- 
dinal course may be the result of a conflation of several intermediate. latent phenotypes that are primarily expressed as neurocognitive or neurophysiological deficit. Identifying the genetic basis of such phenotypes is a daunting, but not impossible, task. Since several genomic regions now appear to be strongly associated with schizophrenia risk. as well as with a variety of alternative phenotypes that may be sharing parts of the same genetic influences, their systematic dissection by convergent genomic and pathophysiological analysis might provide important clues to the causation and genetic architecture of the disorder.

\section{REFERENCES}

Altshuler L.L.. Ventura J., van Gorp W.G., Green M.F., Theberge D.C. \& Mintz J. (2004). Neurocognitive function in clinically stable men with bipolar disorder or schizophrenia and normal control subjects. Biological Psychiatry 56, 560-569.

Arolt V.. Lencer R., Nolte A.. Muller-Myhsok B.. Purmann S. Schurmann M., Leutelt J., Pinnow M. \& Schwinger E. (1996). Eye tracking dysfunction is a putative phenotypic susceptibility marker of schizophrenia and maps to a locus on chromosome $6 \mathrm{p}$ in families with multiple occurrence of the disease. American Journal of Medical Genetics 67, 564-579.

Bearden C.E., Reus V.I. \& Freimer N.B. (2004). Why genetic investigation of psychiatric disorders is so difficult. Current Opinion in Genetics \& Development 14. 280-286.

Bilder R.M., Goldman R.S., Reiter G., Bell L., Bates J.A., Pappadopulos E.. Willson D.F.. Alvir J.M.. Woerner M.G., Geisler S.. Kane J.M. \& Licberman J.A. (2000). Neuropsychology of firstepisode schizophrenia. American Joumal of Psychiatry 157. 549559.

Bleuler E. (1911). Dementia Praecox oder die Gruppe der Schizophrenien. Deuticke: Leipzig. English translation: Dementi Praecox or the Group of Schisophrenias. International Universities Press: New York (1950).

Carpenter W.T.. Heinrichs D.W. \& Wagman A.M. (1988). Deficit and nondeficit forms of schizophrenia: the concept. American Joumal of Psychiatry 145, 578-583.

David A.S., Malmberg A.. Brandt L., Allebeck P. \& Lewis G. (1997). IQ and risk for schizophrenia: a population-based cohort study. Psychological Medicine 27, 1311-1323.

Davidson M., Reichenberg A., Rabinowitz J., Weiser M.. Kaplan Z. \& Mark M. (1999). Behavioral and intellectual markers for schizophrenia in apparently healthy male adolescents. American Journal of Psychiatry 156, 1328-1335.

Egan M.F.,Goldberg T.E., Kolachana B.S.. Callicott J.H., Mazzanti C.M., Straub R.E. Goldman D. \&Weinberger D. (200I). Effect of COMT Vall08/158 Met genotype on frontal lobe function and risk for schizophrenia. PNAS 98. 6917-6922.

Freedman R.. Coon H., Myles-Worsley M.. Orr-Urtreger A., Olincy A.. Davis A.. Polymeropoulos M., Holik J., Hopkins J.. Hoff M. Rosenthal J., Waldo M.C.. Reimherr F.. Wender P.. Yaw J., Young D.A.. Breese C.R.. Adams C.. Patterson D.. Adler L.E.. Kruglyak L., Leonard S. \& Byerley W. (1997). Linkage of a neurophysiological deficit in schizophrenia to a chromosome 15 locus. PNAS 94 $587-592$.

Gottesman I.I \& Bertelsen A. (1989). Confirming unexpressed genotypes for schizophrenia. Archives of General Psychiatry 48:. 867872.
Gottesman I.I. \& Gould T.D. (2003). The endophenotype concept in psychiatry: etymology and strategic intentions. American Journal of Psichiatry 160. 636-645.

Hallmayer J.F. Kalaydjieva L.. Badcock J.. Dragovie M.. Howell S., Michie P.T.. Rock D.. Vile D.. Williams R., Corder E.H., Hollingsworth K. \& Jablensky A. (2005). Genetic evidence for a distinct subtype of schizophrenia characterized by pervasive cognitive deficit. American Joumal of Human Genetics 77. 468-476.

Harrison P. \& Owen M.J. (2003). Genes for schizophrenia? Lancet 361. 417-419.

Harrison P.J. \& Weinberger D.R. (2005). Schizophrenia genes, gene expression, and neuropathology: on the matter of their convergence. Molecular Psichiam 10, 40-68.

Heinrichs R.W. (2004). Meta-analysis and the science of schizophrenia: variant evidence or evidence of variants? Neuroscience and Biobehavioral Reviews 28, 379-394.

Heinrichs R.W. \& Zakzanis K.K. (1998). Neurocognitive deficit in schizophrenia: a quantitative review of the evidence. Neuropsychology 12. 426-445.

Hoff A.L.. Sakuma M.. Wieneke M.. Horon R., Kushner M. \& DeLisi L.E. (1999). Longitudinal neuropsychological follow-up study of patients with first-episode schizophrenia. American Journal of Psychiatry 156, 1336-1341.

Jablensky A., Sartorius N.. Enberg G.. Anker M., Korten A.. Cooper J.E.. Day R. \& Bertelsen A. (1992). Schizophrenia: Manifestations. Incidence and Course in Different Cultures. World Health Organization Ten-Country Study. Psychological Medicine Monograph Supplement 20, 1-97.

Kraepelin E. (1909). Psychiatrie. 8 Auflage. Barth: Leipzig. English translation: Dementia Praecox and Paraphenia (1919). Reprinted by Krieger Publishing Co., Huntington: New York (1971).

Kremen W.S., Buka S.L.. Seidman L.J., Goldstein J.M., Koren D. \& Tsuang M.T. (1998). IQ decline during childhood and adult psychotic symptoms: A 19-year longitudinal study. American Journal of Psychiatry 155, 672-677.

Meehl P.E. (1990). Tow ard an integrated theory of schizotaxia, schizotypy, and schizophrenia. Journal of Personality Disorders 4. 1-99.

Murray C.J.L. \& Lopez A.D. (1996). The Global Burden of Disease. Harvard University Press: Boston.

Paunio T.. Tuulio-Henriksson A., Hiekkalinna T.. Perola M.. Varilo T., Partonen T., Cannon T.D., Lonnqvist J., \& Peltonen L. (2004). Search for cognitive trait components of schizophrenia reveals a locus for verbal learning and memory on $4 \mathrm{q}$ and for visual working memory on 2q. Human Molecular Genetics 13,1693.

Phillips W.A. \& Silverstein S.M. (2003). Convergence of biological and psychological perspectives on cognitive coordination in schizophrenia. Behavioral and Brain Sciences 26, 65-138.

Riley B., Asherson P.J. \& McGuffin P. (2003). Genetics and schizophrenia. In Schizophrenia, 2. ed. (ed. S.R. Hirsch and D. Weinberger), pp. 251-276. Blackwell: Oxford.

Sitskoorn M.M.. Aleman A.. Ebisch S.J., Appels M., Nuyen J. \& Kahn R.S. (2004) Cognitive deficits in relatives of patients with schizophrenia: a meta-analysis. Schizophrenia Research 71, 285-295.

Torrey E.F. (2002). Studies of individuals with schizophrenia never treated with antipsychotic medications: a review. Schizophrenia Research 58, 101-115.

Woodbury M.A., Clive J. \& Garson A. (1978). Mathematical typology: a grade of membership technique for obtaining disease definition. Computers and Biomedical Research 11, 277-298. 